\title{
LA SUCESIÓN NAZARÍ, EL TOPÓNIÑO "GRANADA" Y EL ORIGEN DE SU POBLACIÓN, SEGÚN UN CRONISTA DEL SIGLO XVII
}

\author{
Juan ABELLÁN PÉREZ \\ Universidad de Cádiz
}

\section{Introducción}

La sucesión en el reino Nazarí ha sido abordada, directa e indirectamente, por diversos historiadores ${ }^{1}$, cuya investigación ha conseguido poner orden en la gran confusión que existfa, tal y como se recoge en la Tabla genealógica elaborada por Rachel Arié2. Siendo así,

${ }^{1}$ Seco de Lucena Paredes, L.: sLa familia de Muhammad X el Cojo, rey de Granada» en Al-Andalus, XI (1946), 379-399; «La sultana madre de Boabdil» en Al-Andalus, XII (1947), 359-390; «Un documento del sultán granadino Yüsuf IV Ibn al-Mawl» en Al-Andalus (1948), 497-500; «Una rectificación a la historia de los últimos nasries» en $A l$-Andalus (1952), 153-163; «Nuevas rectificaciones a la historia de los nasríes» en $A l-A n d a l u s, \mathrm{XX}$ (1955), 381-405; «Más rectificaciones a la historia de los nasríes. Un sultán llamado Muhammad el Chiquito* en $\mathrm{Al}$ Andalus (1959), 275-295; Muhammad DX, sultán de Granada, Granada, 1978. Gaspar Remiro, M.:Una rectificación a la geneología de los reyes nazaries de Granada, Granada, 1908. Livermore, H.: «El segundo rey Chico, Muhammad XI y la sucesión de la casa de Abū Nașr Sa d, 1452-1456» en Al-Andalius, (1963), 331-348; Torres Fontes, J.: «Muhammad VIII El Pequeño, rey de Granada» en Miscelánea de Estudios Árabes y Hebraicos, IX (1960), 127-133. López de Coca Castañer, J. E.: «Revisión de una década de la historia granadina», en Miscelánea de Estudios Árabes y Hebraicos, XXIX-XXX (1980-1981). 1973.

${ }^{2}$ Arié, R.: L'Espagne musulmane au temps des nasrides (1232-1492), París, 
alguien pudiera preguntarse el porqué de este trabajo, cuya base documental diverge parcialmente de la realidad. Creo que su justificación está en la edición de una nueva fuente inédita que, como más adelante expondremos, no solamente aporta nuevos datos y posicionamiento de su autor ante la realidad social musulmana sino que tendrá una marcada influencia en autores coetáneos.

\section{El autor y su obra}

El texto que manejamos para este trabajo es un fragmento de una obra más extensa, de autor desconocido, titulada La Corona de Hespania. Historia que tratta de los Reynos, sentorios y estados del Rey de Hespana. Si bien el autor es anónimo, siendo muy dificil su identificación hasta ahora, el manuscrito vierte abundantes datos cronológicos que permite situarlo en la primera mitad del siglo XVII.

El manuscrito se estructura en tres libros. El primero, es un tratado sobre las Leyes de Armeria; en el segundo, se trata de los reinos y señoríos de la Corona de España con sus títulos, ordenados de la siguiente manera: «Rey de Castilla, rey de Leon, rey de Aragon, rey de Napoles, rey de Seçilia, rey de Herusalem, rey de Navarra, rey de Portugal, rey de Galizia, rey de Toledo, rey de Cordoba, rey de Jaen, rey de Sevilla, rey de Valençia, rey de Granada, rey de Malorcas, rey de Sardania, rey de Corsega, rey de Algarves, rey de Algezira, rey de Gibaltar, rey de las islas de Canaria, reyes de las Indias Ocidentales, duque de Milan, duque de Atenas y Neopatria, marques de Oristan y Gosiano, conde de Barçelona, conde de Rosellon y Cerdania, senor de Viscaya, senor de Molina». El tercer libro se dedica a los títulos de «... Austria y Borgonia y de los estados de Flandes ...».

En el libro 2, al tratar del título: Rey de Castilla y Leon, dice "Carlos V), a quien suçedio su hijo don Felipe 2. a quien suçedio el rey don Felipe 3, a quien suçedio su hijo el rey don Felipe 4». Esta misma sucesión se repite en los títulos: Rey de Aragón, Rey de Nápoles y Rey de Sicilia; por tanto, el manuscrito pensamos que fue redactado en el reinado de Felipe IV o al menos el contenido de esos títulos.

El fragmento que analizamos corresponde al libro II, al título: Rey de Granada. Su autor nos informa que la fuente principal en la que se 
basa: «Los reynos de Toledo, Cordoba, Jaen, Sevilla, Valencia, Murçia y Granada, de quien en esta historia escrivo y de quien Vuestra Magestad se intitulo, comenzaron en el tiempo de los moros poseyeron a Hespania, y assy forsamente avre de poner los reyes moros que uvo por aver conquerido con los reyes de Castilla y Leon ...». Todos los reinos que menciona tienen una importante etapa de dominio musulmán, y nos hace la advertencia de que de ordinario los autores yerran a la hora de poner en sus historias los nombres de los reyes moros. Claro está, según su opinión, que no es su caso, ya que él sigue la obra de Luis del Mármol Carvajal, Descripción General de Africa ${ }^{3}$ : «... el qual autor es el mas axçertado de todos los que an escrito de los arabes ...»; ello presupone que nuestro cronista, que hace gala de una gran erudición y manejo de fuentes y bibliografía, conocía otras obras, incluso anteriores a la de Mármol Carvajal, sobre la historia del reino de Granada 4 .

Sin embargo, si comparamos su texto con el de Mármol Carvajal vemos que hay diferencias sustanciales, no sólo respecto al contenido, que en caso de nuestro cronista están justificadas por el carácter de su obra, sino en lo que fundamentalmente hace hincapié, el nombre de los sultanes nazaries y en la omisión de algunos de ellos. En síntesis, el cuadro genealógico del cronista anónimo es más exiguo que el que ofrece Mármol Carvajal.

${ }^{3}$ Mármol Carvajal, Luis del: Descripción General de África, Edición facsímil, Madrid, 1953.

${ }^{4}$ Ladero Quesada, M. A.: «El pasado histórico-fabuloso de España en los nobiliarios castellanos a comienzos del siglo XVIs en Estudios de Historia y de Arqueología Medievales, IX (1993), 55-80, recoge en el apéndice documental un texto titulado: Anpliaçion de la çiudad de Granada, en el que describe la primera parte de la historia de Granada, la prenazarí, con amplia referencia al topónimo. 


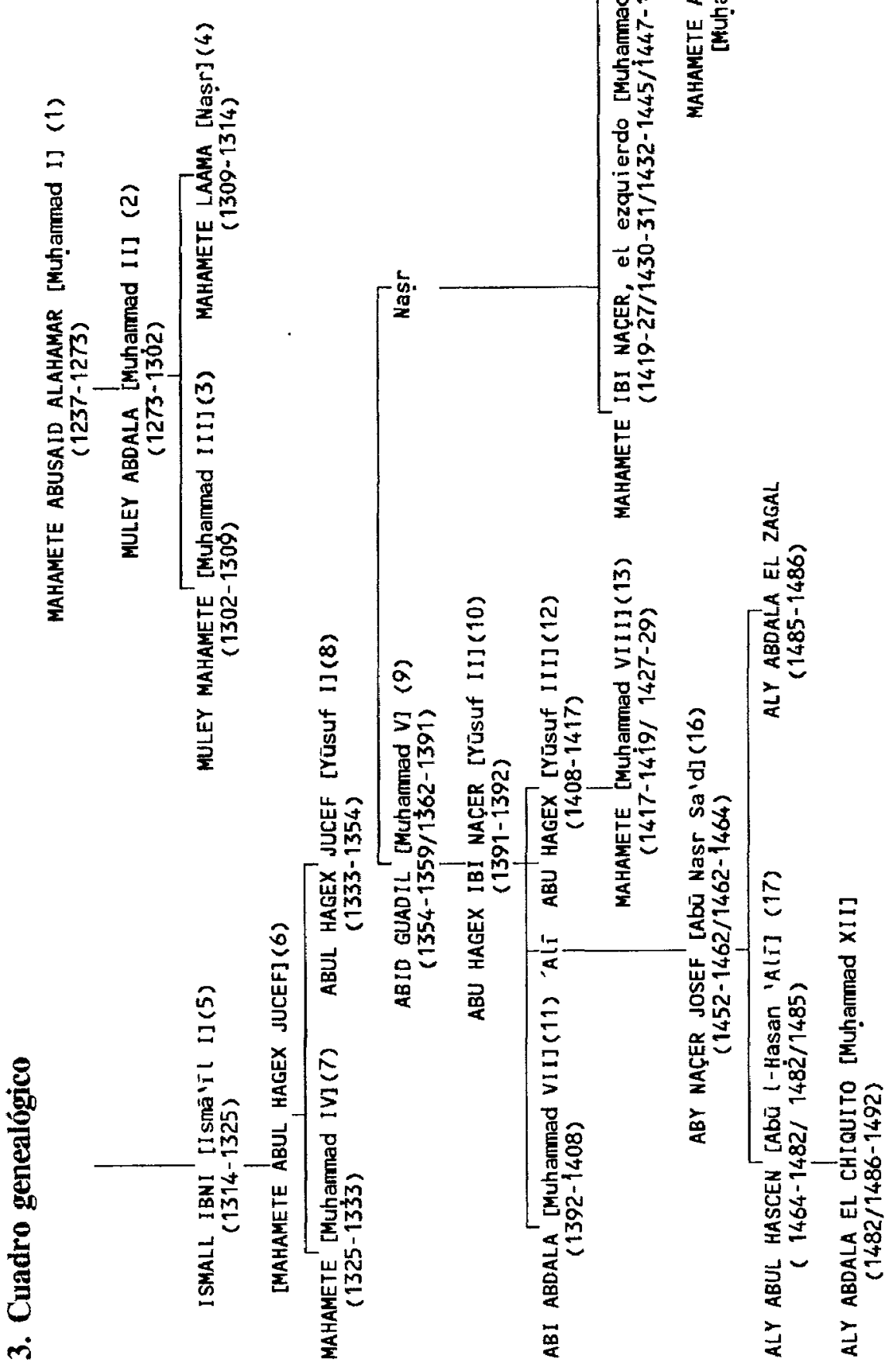




\section{NOTAS AL CUADRO}

(1) Mármol Carvajal lo denomina Mahamete Abu Sayd Ibni Alahamar.

(2) Mármol Carvajal le llama Muley Abdala Aben Mahamete Ibni Nacer; dice que también se llamo Amir el Mocelemin.

(3) Mármol Carvajal: «... se llamo Muley Mahamete, Ibni Aben Alahamar, Ibni Nacer Amir el Mocelemin y por otro nombre le llamaron tambien La ami, que quiere dezir el ciego, mas su nombre proprio era Mahamete ...». Op. cit., 208 r. El inicio de su reinado es correcto en Mármol (1302), sin embargo, no ocurre lo mismo con el final (1312).

(4) Mảrmol Carvajal: «... un hermano de Mahamete rey de Granada, llamado tambien Mahamete, haziendo conjuracion ... se hizo saludar por rey de Granada, y fue quarto rey desta casa de los Aben Alahamares, a quien los Moros llamaron por sobrenombre La Ama (que quiere dezir el ciego) ...». Op. cit., 208 v.

(5) Mármol Carvajal: "... Ismael hijo de Farax alcayde de Malaga, y de una hermana del rey de Granada ... quito el reyno a su tio ... Este lsmael, llamado por sobrenombre Ibni Nacer, fue el primero que reyno por via de hembra en la succession de los reyes de Granada, con el proprio apellido de Aben Alahamar ...». Op. cit., $208 \mathrm{v}$.

(6) El cronista al exponer la sucesión de Ismāin I, dice: «... a quien sucedio su hijo Mahamete Abul Hagex Yucef ...». En principio debía de tratarse de Muhammad IV, sin embargo, el texto que sigue al párrafo anterior: «... en tiempos de este rey uvo en Granada sesenta mill casas, cerco el Albasin y lo dividio de la ziudad, edifico la Alhambra y la torre de Comares, fue vencido juntamente con Abul Haseen, rey de Africa, por el rey don Alonso 11 en la grande vitoria del Salado ...", confirma que se trata de Yüsuf I. Si analizamos el nombre, tal y como aparece en el texto, y sabiendo que a Yũsuf nunca le antecede el nombre de Muhammad podemos aventurar que se trata de una confusión del autor de este texto que por error unió en uno el nombre de los dos sultanes.

(7) A diferencia de nuestro cronista, que funde en un mismo nombre a dos sultanes, Mármol Carvajal dice: «Y como Ismael muriesse ... saludaron por rey a Mahamete Abul Hagex Ibni, que tambien se llamo Ismael Amir el Mocelemin como su padre, y fue sesto rey de la casa de los Alahamares en Granada, y segundo por linea de hembra». Op. cit., $209 \mathrm{v}$.

(8) Mármol Carvajal: «... los Moros de Granada alçaron luego por Rey a un hijo suyo llamado Abu Abdeli Abul Hegeg Iucef ..... Op. cit., 210 v. Aunque la cronología que ofrece sobre este reinado es exacta, no lo es el parentesco, ya çue Yūsuf I no era hijo de Muhammad IV sino hermano.

(9) La sucesión de Yūsuf I es correcta. El cronista dice: «Sucediole su hijo Abid Guadil ...»; sin embargo, omite los reinados de Ismän I (1314-1325) y de 
Muḥammad VI el Bermejo (1360-1362), quizá porque los consideraba usurpadores. Este cronista se limita a mencionar al segundo con el título de rey al exponer que se levantó contra Muḥammad V: «... Mahamete el Bermejo, rey a quien el rey don Pedro hizo matar en Sevylla ...». Como acabo de expresar, El Bermejo no aparece en esta sucesión de la casa de Granada. A diferencia de la fuente en que se basa nuestro cronista, que afirma que a Yūsuf I le sucedió «... Abil Guadid Ibni Nacer, su tio: hermano de su padre, que tambien se intitulo Abu Abdeli Amir el Mocelemin ...», op. cit., 214 v., la suya es correcta, ya que Abil Guadid (Muhammad V) no era hijo de Muhammad IV, sino de Yūsuf I. Como ocurre con nuestro cronista, Mármol Carvajal omite el brevísimo reinado de Ismācil II que, como se sabe, se inició en 1359; sin embargo, Mármol da esa fecha como la del levantamiento e inicio del reinado de Muhammad VI el Bermejo contra Muhammad V: «... en el año de mil y trezientos y cinquenta y nueue, se leuanto contra el otro Moro de la casa de los Alahamares llamado Mahamete ...». Op. cit., 214. v., y volvió a reinar por segunda vez en 1362: «Sabida la muerte de Mahamete en Ronda, Abil Guadid fue a Granada, y los ciudadanos lo rescibieron luego por rey, y por que era ya viejo, y auia reynado otra vez, le llamaron Muley Xeques.

(10) Mármol Carvajal: «Murio tambien Abil Guadid rey de Granada, succediole un hijo suyo llamado Abul Hagex Ibni Nacer, el qual se apodero pacificamente del reyno ..... Op. cit., 217 r.

(11) Muḥammad VII aparece citado por su kunya Abū ${ }^{\circ} A b d$ Allăh. El cronista dice sobre este sultán, 12 de la sucesión según la tabla genealógica de R. Arié, 11 según Mármol Carvajal y 10 en nuestro texto, que murió «... en una marlota y muy rica atosinada que le enbio el rey de $\mathrm{Fez}$ (Abu Sa ${ }^{\circ} \mathrm{id}$ III), $y$ vistiendosela se le cayeron las carnes apedazoss. Esta noticia la hemos encontrado en $\mathrm{La}$ «Historia de la Casa Real de Granada». Anónimo castellano de mediados del siglo XVI, pág. 173 : «... sucedió la muerte de Mahomad, de una camisa entosigada que se puso, a los 12 años de su reyno, el de 1408 ...»; sin embargo, Mármol Carvajal, que tambien la recoge de la siguiente manera: «... en el año del señor de mil y trezientos y nouenta y seys, Abu Henun rey de Fez teniendo odio secreto con Abi Abdala rey de Granada, y queriendole hazer morir, en le embio un rico presente de jaezes, y vestidos, y entre las otras cosas, fue una marlota teñida, y texida con ponçoña, y como fuesse muy rica, y el Moro se la vistiesse, murio dentro de treynta dias, cayendose le las carnes a pedaços, succediole Mahamete su hijo ...», op. cit., 217 v., yerra en la fecha de la muerte y en la sucesión al confundir Abi Abdala y Muhammad (éste último murió según él en 1409), como dos sultanes, cuando Abi Abdala fue la kunya de Muhammad y por tanto el mismo sultán, Muhammad VII.

(12) Mármol Carvajal: «Boluiendo pues a Mahamete rey de Granada ... adolescio de enfermedad, y dizen los escriptores Arabes, que ... teniendo preso en el 
castillo de Salobreña a un hermano suyo llamado Abul Hagex ... fue Abul Hagex saludado luego por rey, el qual tomo pacificamente possession de todo el reyno, y fue el trezeno de la casa de los Alahamares». Op. cit., 219 v.

(13) El autor del texto vuelve a equivocarse, ahora, respecto a la sucesión de Muhammad VII. Dice que le sucedió su hijo Mahamete cuando se sabe perfectamente que a su muerte, acaecida el 13 de mayo de 1408, subió al trono de la Alhambra su hermano Yūsuf III, que estaba prisionero en la cárcel de la fortaleza de Salobreña. El Mahamete al que se refiere el texto no era su hijo, sino de su hermano Yūsuf III, por tanto, el futuro Muhammad VIII al-Sagìr o El Pequeño. (14) El cronista dice, respecto a la sucesión de Yüsuf III : «Sucediole su hijo Mahamete ibi Naçer, el esquierdo». Como ya es sabido y está perfectamente establecido, Muhammad IX al-Aysar (Cfr. Seco de Lucena Paredes, L.: Muhammad IX, sultán de Granada, Granada, 1978) no era hijo de Yūsuf III sino primo hermano, y ambos, nietos de Muhammad V. A Yūsuf III no le sucedió su primo, el Zurdo, sino su hijo Muhammad VIII al-Sagir y a éste Muhammad IX, hijo del infante Nașr. Mármol Carvajal, por su parte, cae en el mismo error que nuestro cronista al afirmar que: «Murio el rey Abul Hagex, en este año de mil y quatro cientos y veynte y tres (este acontecimiento ocurrió en 1417). Succedio le Muley Mahamete el Azeri su hijo (a quien llamaron tambien Ibni Nacer Aben Abul Hagex Amir el Mocelemin y los Christianos le llamaron el rey yzquierdo ..... Op. cit., $220 \mathrm{v}$. El primer reinado de El Zurdo llega hasta el año 1427 y dio paso al segundo y último de Muhammad VIII. Mármol Carvajal omite el primer gobierno de Muhammad VIII, sin embargo, posteriormente recoge como primero su segundo acceso al poder: "Leuanto se en este tiempo en el reyno de Granada contra Muley Mahamete Ibni Nacer un primo suyo llamado Muley Mahamete el çaguer, y siendo muy fauorecido del pueblo le echo del reyno, en el año de mil y quatrocientos y veynte y siete ...*. Op. cit., $231 \mathrm{r}$. Estos reinados partidos, debidos a sucesivos golpes de estado, no se recogen en la fuente que manejamos. Tras el segundo gobierno de Muhammad IX, muy breve (1430-31) (Mármol Carvajal, op. cit., 221 r.) fue entronizado Yūsuf IV (1431-32), sultán que no se recoge en nuestro texto y sí en el de Mármol Carvajal: «... Iucef Aben Muley nieto del rey Moro que el rey Don Pedro mato en Seuilla (Muhammad el Bermejo)...». Op. cit., 221 v. De nuevo vuelve al poder El Zurdo (1432-1445). Este tercer gobierno también lo recoge Mármol: «... los de Granada embiaron a llamar a Mahamete Ibni Nacer que estaua en Malaga, $y$ le recibieron otra vez por rey ..... Op. cit, 221 v.

(15) A Muhammad IX, según el cronista: «Sucediole Mahamete Aben Odman ...», es decir, el hijo de su hermano Utman, Muhammad X al-Afnah (1445); sin embargo, todavía habría de volver el Zurdo. Mármol Carvajal dice: "Reynando pues en Granada Mahamete Ibni Nacer, que con tantas coçobras posseyo aquel reyno, en el año de mil y quatrocientos y quarenta y cinco, se leuanto contra el 
un sobrino suyo, hijo de Odman su hermano, que ya era muerto, llamado Muley Mahamete Ibni Odman ...». Op. cit., 223 r. A éste le sucedió Yüsuf V (14451446) que no es recogido en nuestro texto y sí por Mármol Carvajal: $\ll_{. .}$otro Infante Moro llamado Ismael, hijo de Aben Muley (Ahmad, hijo de Yüsuf II), el aspirando a la succession del reyno, mouio luego guerra a Mahamete Ibni Odman ...m. Op. cit., 223 r. Muhammad X el Cojo volví́ al poder en un segundo gobierno que duró de 1446-1447. Tras este sultán se inicia el último gobierno de El Izquierdo (1447-1453/4). Durante este último gobierno de Muhammad IX fue asociado al trono Muhammad XI el Chiquito (1451-51/1453-4) pero tampoco es recogido por nuestro cronista ni por Mármol.

(16) El autor de nuestro texto, como hemos indicado, no recoge los sultanes que se intercalan en los cuatros gobiernos de Muhammad IX y da como su sucesor directo a Muhammad X. A éste según el citado cronista le sucedió «... su primo Abu Naçer Josef que fue vasallo del rey don Enrico 4, y le pagava dose mill doblas de tributo cada un año ...». Es cierto que Abū Nașr $\mathrm{Sa}^{c} \mathrm{~d}$ era primo de Muhammad X pero no fue su sucesor directo. En el mismo error cae Mármol Carvajal: «... un primo de Aben Odman llamado Abi Nacer Abdilehi, que tambien se llamo Iucef, se alço contra el, y le desposseyo del reyno ...*. Op. cit., $223 \mathrm{v}$. (17) El resto de la sucesión hasta la caída del reino de Granada en poder de los Reyes Católicos responde correctamentea la establecida por otros autores. A Abū Nașr $\mathrm{Sa}^{\mathrm{c}} \mathrm{d}$ le sucedió su hijo Ali Abul Hascen [Abūl Hasan 'A$\overline{1}$ ] y a éste su hijo Aly Abdala, el Chiquito, el Logoibe > Zugubi, 'El desventuradillo' que «... fue preso junto a Lusena por el conde de Cabra y el alcayde de los Donzeles, capitanes de los Reyes Catolicos ...*; contra el que se alzó su tío: «... los moros de Granada alçaron por rey a su tio Aby Abdala llamado el Zagal .... y de nuevo Muhammad XII (Boabdil): «... mas puesto en libertad por los Reyes Catolicos, el rey Chiquito tuvo con su tio guerra sobre el reyno, y al fyn bolvio a reynar en Granada ...».

\section{El topónimo Granada}

$\mathrm{El}$ autor del texto recoge, tomando de las historias de los árabes que manejo, dos de las muchas definiciones que se han dado al nombre de Granada. La primera es quizá la más conocida; según sus lecturas, Granada, en torno al año mil, no estaba muy poblada y se le llamaba Hisma Roman = 'la villa de Granada'; sin embargo, el topónimo debió 
de ser Hiṣn al-Rummän, 'el castillo de las Granadas'5; no obstante, la segunda acepción, en nuestra opinión, es la más original y fantástica, al hacer venir el nombre de Granada del vocablo Gar, 'cueva' y de Nada; este último término, se aplica a un grupo étnico originario de la ciudad de Nada de las Palmas que estaba situada en la sierra de Damasco, y que llegaron a la Península acompañando a las tropas de Tarif; por tanto, según esta teoría, que no hemos documentado en ningún otro estudio, Granada evolucionó a partir de la conjunción de los dos vocablos apuntados Gar y Nada o Nata, dando Garnada o Garnata.

\section{Sobre el origen de la población granadina}

Un aspecto muy interesante que, sin duda, a nuestro juicio, merece la pena destacar es la justificación que el autor del texto que publicamos hace de la conquista de Granada por los Reyes Católicos. La base de su argumentación se remonta al siglo XIV, a la petición que Jaime II de Aragón hiciera al pontúfice Clemente $\mathrm{V}$ en el Concilio Universal de Viena (1311), solicitándole la concesión de «... el suçedio de las desimas exclesiasticas ...» para ayudar a la guerra contra el Islam peninsular. El monarca aragonés, para mover la voluntad del Papa, le informó que de las 200.000 personas que vivían en la ciudad de Granada no se hallaban «... quinientos que fuese moros naturales, que no tuvieran padres o abuelos cristianos, y avia sinquenta mill personas que renegaron la fe de Chripto, y pasavan de treynta mill cristianos los que estavan cautivos ...". Estas cifras que recoge nuestro cronista son, evidentemente, erróneas si las aplicamos como él hace a la ciudad de Granada, incluso, si incluyéramos en ella El Albaicín, La Alhambra, los arrabales y las alquerías de la Vega que en los años próximos a la conquista por los Reyes Católicos no superaban los 50.000 habitantes; sin embargo, esa población tiene visos de realidad si se refiriera a la totalidad del Reino a comienzos del siglo XIV. M. A. Ladero, por su

${ }^{5}$ Simonet, F. J.: Descripción del Reino de Granada, sacada de los autores arábigos, 711-1492, Granada, 1872 (Amsterdam, 1979), 32 y 42, recoge que Hisn Arroman, junto con Casthella y Granata, era una fortaleza de la llíberis situada en la Alcazaba del Albaicín. 
parte, cifra la población del reino nazarí a finales del siglo siguiente en 300.000 personas 6 .

Aceptando como válida la población que ofrece el cronista pero para todo el reịno nazari, se plantea el origen hispánico de la mayoría de la población, es decir, la continuidad del poblamiento sobre el solar granadino, estimado por nuestro cronista en un $99,75 \%$ que distribuye de la siguiente manera:

No hispanos

Hispanos:

Muladíes

Renegados

Cautivos
500

119.500

50.000

30.000
$0,25 \%$

$59,75 \%$

$25,00 \%$

$15,00 \%$

De nuevo nos encontramos al analizar este cuadro con unos porcentajes excesivos, con una desproporción entre los dos bloques, puesto que sería mayor el número de no hispanos y, en consecuencia, menor el de los hispanos. Al margen de esta cuantificación, hay algo de verdad; es indudable que desde el mismo momento de la conquista, los hispanovisigodos eran mayoría frente al grupo dominador, asi como su conversión masiva a la nueva religión -muladfes-. A este grupo que encontramos en la Granada prenazari, se añaden sucesivamente otros contingentes, de igual origen, conforme se van produciendo los avances reconquistadores de la Corona de Castilla. Si a ellos unimos los nuevos conversos o renegados y los cautivos, en cifras inferiores a las apuntadas en el cuadro, está claro que se cumple lo que apunta el

${ }^{6}$ Ladero Quesada, M. A.: «Datos demográficos sobre los musulmanes de Granada y Castilla en el siglo XV” en Anuario de Estudios Medievales, 8 (19721973), 486. Un espléndido estudio sobre la población puede verse en Peinado Santaella, R. G. y López de Coca Castañer, J. E.: Historia de Granada. II: La época medieval (Siglos VIIl-XV), Granada, 1987, 308-310; Guichard, P.: AlAndalus. Estructura antropológica de una sociedad islámica de Occidente, Barcelona, 1986; Bosch Vilá, J.: «El elemento humano norteafricano en la Historia de la España musulmanas, en Cuadernos de la Biblioteca Española de Tetuán, 2 (1964); Simonet, J. F.: Influencia del elemento indigena en la cultura de los moros del reino de Granada, Tánger, 1895. 
cronista de que la inmensa mayoría de estos individuos tenía un antecesor cristiano.

En el extremo opuesto, el de los no hispanos, nos parece tan ridícula la cifra que ofrece el texto como excesiva la afirmación de Ibn al-Jațib de que la población de Granada era mayoritariamente de origen árabe ${ }^{7}$; aunque no tenemos datos que nos lo puedan confirmar, sabemos que al grupo de los árabes se unen los beréberes, zanatas y sinhayas. ¿En qué proporción?. No se sabe, aunque las sucesivas oleadas del Norte de África darían una superioridad a los segundos ${ }^{8}$; sin embargo, es curioso que nuestro cronista no hiciera ninguna alusión a los judíos que habitaban en el reino de Granada, ni siquiera a los de la ciudad, a quienes se atribuye la fundación de Garnata.

El planteamiento que hace el cronista, dando un predominio casi absoluto al origen hispano de la población nazarí, no tiene otro sentido que el de justificar, dentro de su concepción cristiana de la Historia, la intervención de los Reyes Católicos, sin que se haga ninguna alusión al problema que planteó la población mudéjar y su conversión forzosa al cristianismo.

\section{Apéndice}

\section{REY DE GRANADA}

Granada como se lee en las Historias de los arabes, cerca de los años de mill no era muy grande su poblaçion y la llamavan los moros Hisma Roman que quiere dizir la villa de Granada, de donde pareçe que de este arbol tomo el nombre, otros dizen que ally avia una cueva que los moros tuvieron en veneraçion, y porque llaman a la cueva Gar y este lugar fue poblado de unos moros que pasaron con Tarif en Hespania, naturales de Nada de las Palmas, ziudad de la çierra de

${ }^{7}$ Véase Peinado Santaella, R. G. y López de Coca Castañer, J. E.: Historia de Granada, op. cit., 310.

8 Este punto ha sido abordado especialmente por el Dr. Bosch Vilá «Andalucía islámica: arabización o berberización. Apuntes y reflexiones en torno a un viejo tema» en Andalucía Islámica, I (1980). 
Damasco, juntando ambos terminos Gar y Nata vinieron los moros a llamar aquella poblaçion, y agora Granada; viene despues a mayor grandesa quando los moros perdieron el reyno de Cordoba que gano el rey don Fernando el Santo el año de 1236 porque alçandose algunos alcaydes moros con las ziudades que podian Mahamete Abusaid Alahamar que era un valeroso capitan, alcayde de Arjona se hizo rey y le obedisieron Guadiz, Baeça y Jaen y ultimamente la ziudad de Granada donde puso la silia real, y estableçio su reyno; el año de 1237, fue vasallo del rey don Fernando el Santo.

Sucediole su hijo Muley Abdala que fue vasallo del rey don Alonso el Sabio.

Sucediole su hijo Muley Mahamete.

Sucediole su hermano Mahamete La ama.

Sucediole su sobrino Ismall ibni, a quien sucedio su hijo Mahamete Abul Hagex Juçef, en tiempo de este rey uvo en Granada sesenta mill casas, cerco el Albisin y lo dividio de la ziudad, edifico el Alhambra y la torre de Comares, fue vençido juntamente con Abul Haseen rey de Africa por el rey don Alonso 11 en la grande vitoria del Salado, donde los escritores dizen que murieron dosientos mill moros.

Sucediole su hijo Abid Guadil, contra este se levanto Mahamete el Bermejo, rey a quien el rey don Pedro hizo matar en Sevylla, y quedo Abid Guadil pacifico en su reyno de Granada y se hizo vasallo del rey don Pedro.

Sucediole su hijo Abut Hagex ibi Naçer.

Sucediole su hijo Abi Abdala, murio en una marlota y muy rica atoçiçada que le embio el rey de $\mathrm{Fez}$, y vistiendosela se le cayeron las carnes apedasos.

Sucediole su hijo Mahamete.

Sucediole su hermano Abul Hager.

Sucediole su hijo Mahamete ibi Naçer, el esquierdo. Sucediole Mahamete Aben Odman, a quien sucedio su primo Aby Naçer Josef que fue vasallo del rey don Enrico 4, y le pagava dose mill doblas de tributo cada un año.

Sucediole su hijo Aly Abul Hasçen.

Sucediole su hijo Aly Abdala el Chiquito llamado el Logoibe que fue preso junto a Lusena por el conde de Cabra y el alcayde de los 
donzeles, capitanes de los Reyes Catolicos, y entonçes los moros de Granada alçaron por rey a su tio Aby Abdala llamado el Zagal mas puesto en libertad por los Reyes Catolicos, el rey Chiquito tuvo con su tio guerra sobre el reyno y al fin bolvio a reynar en Granada, y los Reyes Catolicos aviendo començado la guerra de aquel reyno el año de 1482 le acabaron de conquistar ganando ultimamente la ziudad de Granada el año de 1492 que fue vitoria muy celebre en el mundo y se intitularon de ally adelante reyes de Granada, le pusieron en el escudo real la granada por armas y blason del reyno que avian conquistado, con lo qual se libro aquella prostera parte de Hespania de la cervidumbre de los moros, y se puso fin a la contienda y guerra de ochocientos años, lo que no se sabe que aya durado tanto tiempo entre reyes tan vezinos y contra enemigos tan valientes y exercitados, y quan emportante fue a Hespania la conquista de este reyno se entendera por lo que el rey don Jaime 2 de Aragon pidio al papa Clemente 5 estando en el consilio universal de Viena el año de 1311 que para ayuda de las guerras que tenia contra los moros del reyno de Granada le dize el suçedio de las desimas ecleziasticas y por mover el pontifiçe le informava que en la ziudad de Granada avia dosientos mill personas y no se hallavan quinientos que fuese moros naturales que no tuviesen padres o abuelos cristianos, y avia sinquenta mill personas que renegaron la fe Chripto, y pasavan de treynta mill cristianos los que estavan cautivos, esta empresa tan cristiana y santa se guardo para estos bienaventurados reyes que por ella y otras esclaresidas hazañas que hisieron alcasaron el renombre de Catolicos y son la honra y gloria de los reyes de Hespania. 